\title{
Combining alpha radiation-based brachytherapy with immunomodulators promotes complete tumor regression in mice via tumor-specific long-term immune response
}

\author{
Vered Domankevich ${ }^{1} \cdot$ Adi Cohen $^{1} \cdot$ Margalit Efrati $^{1} \cdot$ Michael Schmidt $^{2,3} \cdot$ Hans-Georg Rammensee $^{4} \cdot$ Sujit S. Nair $^{5}$. \\ Ashutosh Tewari ${ }^{5} \cdot$ Itzhak Kelson $^{2,3} \cdot$ Yona Keisari ${ }^{1,3}$
}

Received: 5 March 2019 / Accepted: 10 October 2019 / Published online: 22 October 2019

(c) The Author(s) 2019

\begin{abstract}
Diffusing alpha-emitters radiation therapy (DaRT) is the only known method for treating solid tumors with highly destructive alpha radiation. More importantly, as a monotherapy, DaRT has been shown to induce a systemic antitumor immune response following tumor ablation. Here, immunomodulatory strategies to boost the antitumor immune response induced by DaRT, and the response specificity, were investigated in the colon cancer CT26 mouse model. Local treatment prior to DaRT, with the TLR3 agonist poly I:C, was sufficient to inhibit tumor growth relative to poly I:C or DaRT alone. DaRT used in combination with the TLR 9 agonist CpG, or with the TLR $1 / 2$ agonist XS15 retarded tumor growth and increased tumor-rejection rates, compared to DaRT alone, curing $41 \%$ and $20 \%$ of the mice, respectively. DaRT in combination with $\mathrm{CpG}$, the Treg inhibitor cyclophosphamide, and the MDSC inhibitor sildenafil, cured 51\% of the animals, compared to only $6 \%$ and $0 \%$ cure when immunomodulation or DaRT was used alone, respectively. Challenge and Winn assays revealed that these high cure rates involved a specific immunological memory against CT26 antigens. We suggest that DaRT acts in synergy with immunomodulation to induce a specific and systemic antitumor immune response. This strategy may serve as a safe and efficient method not only for tumor ablation, but also for in situ vaccination of cancer patients.
\end{abstract}

Keywords Tumor ablation $\cdot$ Poly I:C $\cdot \mathrm{CpG} \cdot \mathrm{XS} 15 \cdot$ Treg $\cdot$ MDSC

\section{Abbreviations}

$\mathrm{Bq} \quad$ Becquerel

CP Cyclophosphamide

DAMP Damage-associated molecular pattern

DaRT Diffusing alpha-emitters radiation therapy

Ra Radium

STR Short Tandem Repeat

Note on previous publication Part of this work was presented as a poster at the 33rd Annual Meeting of the Society for Immunotherapy of Cancer (SITC), Washington D.C, USA, Nov. 7-11, 2018; the 8th conference of the International Cancer Microenvironment Society (ICMS), Lisbon, Portugal, June 10-14, 2018; the EACR-AACR-ISCR Conference, Jerusalem, Israel, Oct. 9-11, 2018; European Society for Radiotherapy and Oncology (ESTRO) 2018, Barcelona, Spain, 20-24 April, 2018.

Vered Domankevich and Adi Cohen have contributed equally to this paper.

Yona Keisari

ykeisari@tauex.tau.ac.il

Extended author information available on the last page of the article

\section{Introduction}

Ablation strategies are non-surgical procedures that destroy solid tumors in situ, thereby releasing tumor antigens and damage-associated molecular pattern (DAMP) molecules, which then promote a systemic antitumor immune response (for review, see [1]). A unique method for solid tumor ablation using highly destructive alpha radiation was developed in our laboratories, termed diffusing alpha-emitters radiation therapy (DaRT) $[2,3]$. The treatment was found to destroy mouse and human tumors of squamous cell carcinoma [4, 5], lung carcinoma [6], prostate, glioblastoma, colon [7], and pancreatic [8] tumors. By widening the range of the alphaemitting atoms' distribution inside the tumor, this method provides the only known application for alpha-based brachytherapy of solid tumors.

Synergy between radiotherapy and immunotherapy has been demonstrated in preclinical trials, and a mechanistic rationale to combine both therapies has been suggested. Radiation increases antigen visibility, enhances MHC1 expression, and promotes phagocytosis by 
antigen-presenting cells, thereby leading to T-cell priming, antigen-specific recognition and, in some cases an abscopal effect [9]. Specifically, particle radiation, such as proton and carbon ions, induces an antitumor response, resulting in the suppression of distant metastases [10]. However, immune activation by radiation is strictly regulated to prevent unwanted recognition of self-antigens, for example, by recruitment of MDSCs to the radiated site [9]. Thus, methods to boost antitumor immunity induced by radiation include strategies to counteract the immunosuppressive tumor microenvironment. Another possibility is to stimulate the immune response induced by radiotherapy using immunoadjuvants that may activate dendritic cells and promote antigen cross-priming [11].

Tumor ablation by DaRT has been shown to induce a systemic antitumor immune response against tumor cells. In the colorectal carcinoma CT26 mouse model, DaRT protected against tumor challenge in both the skin and lungs, and in the murine mammary cancer model, DA3, DaRT protected against skin tumor challenge and inhibited lung metastases [12]. Furthermore, DaRT-mediated systemic antitumor immune response is significantly enhanced when combined with immunomodulators. In the DA3 breast cancer model, DaRT combined with the TLR9 agonist $\mathrm{CpG}$ further retarded tumor growth compared to each treatment alone [12]. Combining DaRT with Treg/ MDSC inhibitors protected against primary/challenge tumor development [13], whereas DaRT combined with $\mathrm{CpG}$ and Treg/MDSC inhibitors reduced lung metastases and enhanced the tumor response, leading to complete tumor rejection.

In the present study, we used the colon CT26 tumor model to investigate two main scientific questions: (1) Can other types of TLR agonists be used in combination with DaRT to stimulate antitumor immunity? [2] What is the nature and specificity of the immune response triggered by DaRT when combined with immunostimulation and inhibition of suppressor immune cells?

\section{Materials and methods}

\section{Animals}

$\mathrm{BALB} / \mathrm{c}$ female mice $(\sim 20 \mathrm{~g}, 10$ weeks old $)$ were kept in the animal facility at Tel Aviv University. All surgical and invasive procedures were performed under anesthesia using ketamine (100 mg/kg, Bremer Pharma, Germany) and xylazine hydrochloride $(10 \mathrm{mg} / \mathrm{kg}$, Eurovet Animal Health B.V., Bladel, Netherlands) solution in phosphatebuffered saline (PBS). An i.p. injection was given $10 \mathrm{~min}$ before starting the treatment.

\section{Tumor cell lines}

All cell lines were stored in a humid incubator at a temperature of $37^{\circ} \mathrm{C}$ and $5 \% \mathrm{CO}_{2}$. CT26 cells were grown in RPMI-1640 containing L-glutamine, supplemented with $10 \%$ fetal calf serum, penicillin $(100 \mathrm{U} / \mathrm{ml})$, streptomycin $(100 \mu \mathrm{g} / \mathrm{ml})$, nystatin $(12.5 \mathrm{U} / \mathrm{ml})$, sodium pyruvate $(1 \mathrm{mM})$, and HEPES buffer (1 M) (Biological Industries, Kibbutz Beit Haemek, Israel).

DA3 cells were grown in Dulbecco's modified Eagle's medium containing $4.5 \mathrm{~g} / \mathrm{l} \mathrm{D}$-glucose and $4 \mathrm{mM} \mathrm{L-glu}$ tamine, supplemented with $10 \%$ fetal calf serum, penicillin $(100 \mathrm{U} / \mathrm{ml})$, streptomycin $(100 \mu \mathrm{g} / \mathrm{ml})$, and nystatin (12.5 U/ml) (Biological Industries).

\section{Tumor cell inoculation}

Mice were inoculated i.d. with $5 \times 10^{5}$ cells, unless otherwise specified, into the low lateral side of the back in $0.05 \mathrm{ml}$ RPMI or Hanks' balanced salt solution (HBSS) (Biological Industries).

\section{Tumor volume measurements}

Local tumor growth was determined by measuring three mutually orthogonal tumor dimensions two to three times per week, according to the following formula: Tumor volume $=\pi / 6 \times$ diameter $1 \times$ diameter $2 \times$ height. For cumulative data of two or more experiments, extrapolation using the TREND function in Excel (based on the two closest existing measurements) was used in the case of missing corresponding measurement time points.

\section{Immunomodulators preparation}

CpG (Syntezza, Jerusalem, Israel) was dissolved in PBS to the indicated concentrations. PBS served as the control. Cyclophosphamide (CP) (Sigma, Israel) was dissolved in saline. Saline served as the control. Sildenafil (Pfizer, NY) was prepared as previously described [14]. Glass bottles were used to avoid absorption and bottles were covered with aluminum foil to protect from the light. Bottles were shaken five times a week, and the solution was exchanged twice a week. XS15 (N-palmitoyl-S-[2,3bis(palmitoyloxy)-(2R)-propyl]-(R)-cysteinyl-GDPKHPKSF) (EMC Microcollections, Tübingen, Germany) [15] was dissolved in sterile endotoxin-free water according to the manufacturer's instructions. High-molecularweight poly I:C (InvivoGen, Toulouse, France) was dissolved in PBS or physiological water according to the 
manufacturer's instructions. All of the reagents for injection were prepared using sterile solutes in a biohazard hood.

\section{Winn assay}

Spleens were harvested, immersed in PBS, ground with the flat end of a syringe and passed through a cell strainer. Cells were washed in RPMI/HBSS and centrifuged at $394 g$ for $7 \mathrm{~min}$. The supernatant was removed, and cells were resuspended and pooled. Red blood cells were lysed, and cells were washed in HBSS. Cells were then mixed with tumor cells in the indicated concentrations and immediately injected in a volume of $0.2 \mathrm{ml}$.

\section{${ }^{224}$ Radium (Ra)-loaded seed (DaRT seed) preparation and insertion}

Stainless steel wires ( $0.4 \mathrm{~mm}$ diameter, $6-8 \mathrm{~mm}$ length) were loaded with ${ }^{224} \mathrm{Ra}$ atoms (half-life of 3.7 days). To prevent Ra dissolution in the tissue fluids, the atoms were embedded a few atomic layers into the seed surface through thermal treatment [2]. Seeds, either loaded with ${ }^{224} \mathrm{Ra}$ or inert, were placed near the tip of a 21-gauge needle attached to an insertion applicator. The radioactive and inert seeds were inserted into the primary tumor under anesthesia.

\section{Statistical analysis}

Statistical significance ( $p$ value) was determined by twotailed Student's $t$ test for comparisons of group means or by $\chi^{2}$ test for comparisons of proportions between experimental groups.

\section{Results}

\section{Combined treatment with DaRT and the TLR3 agonist poly I:C retards $\mathrm{CT} 26$ tumor development compared to each treatment alone}

The effect of DaRT combined with the TLR3 agonist poly $\mathrm{I}: \mathrm{C}$ on tumor development was investigated in the immunogenic CT26 tumor model. The poly I:C $(10 \mu \mathrm{g} / 30 \mu \mathrm{l})$ was injected intratumorally into CT26-bearing mice $72 \mathrm{~h}$ and $24 \mathrm{~h}$ prior to DaRT treatment. When tumor maximal length reached $7.4 \mathrm{~mm}$, a $7-\mathrm{mm} 40 \mathrm{kBq}$ DaRT seed was inserted into the tumor (Fig. 1a). DaRT and local poly I:C treatments were sufficient to significantly retard tumor growth compared to all other groups (Fig. 1b) $\left(p_{t \text { test }}<0.05\right.$ for DaRT + poly I:C vs. control on days 3, 13, 14, DaRT alone on days $4-8$, and poly I:C alone on days $3,8-10,12,13$ ). Notably, DaRT alone significantly retarded tumor growth $\left(p_{t \text { test }}<0.05\right)$ on days 13 and 14 , whereas poly I:C alone was not significantly different from the control at any of the time points.

\section{Combined treatment with DaRT and the TLR9 agonist CpG, or the TLR1,2 agonist XS15, retards CT26 tumor development and increases tumor-rejection rates compared to DaRT alone}

The above results agreed with our previous findings in the DA3 breast cancer model showing that DaRT in combination with the TLR9 agonist CpG significantly inhibits tumor development compared to each of the treatments alone [12]. We also confirmed the enhancement of DaRTinduced tumor retardation by $\mathrm{CpG}$ in the $\mathrm{CT} 26$ tumor model. In addition, potential enhancement by the TLR1,2 agonist XS15 was examined. CT26-bearing mice were treated with a $100 \mu \mathrm{g} / 30 \mu \mathrm{l}$ peritumoral injection of $\mathrm{CpG}$ immediately prior to DaRT insertion, and then a dose of $20 \mu \mathrm{g} / 10 \mu \mathrm{CpG}$ administered intranasally three times (once every 2 days), starting 3 days after DaRT insertion, or with a $40 \mu \mathrm{g} / 50 \mu \mathrm{l}$ peritumoral injection of XS15 once a week for 3 weeks from the day of DaRT insertion (Fig. 2a). A 7-mm-long DaRT seed $(40-50 \mathrm{kBq})$ was inserted into the tumor when its maximal length reached $8-10 \mathrm{~mm}$.

DaRT combined with either CpG or XS15 treatment retarded tumor development compared to DaRT alone. Tumor volume on day 10 for mice treated with DaRT was $738 \pm 170 \mathrm{~mm}^{3}$; with DaRT + XS15: $252 \pm 39 \mathrm{~mm}^{3}$; and for DaRT + CpG: $187 \pm 61 \mathrm{~mm}^{3}\left(p_{t \text { test }}<0.05\right.$ on days 6 and 10$)$. In addition, DaRT combined with CpG or XS15 cured 5 out of $12(41 \%)$ or 2 out of $10(20 \%)$ of the mice, respectively. The cure rates for animals treated with DaRT combined with $\mathrm{CpG}$ were significantly different from those treated with DaRT alone (0\% cure; $P_{\chi 2 \text { test }}<0.05$; Fig. 2 b).

\section{Combined treatment with DaRT, CpG, $\mathrm{CP}$, and sildenafil inhibits $\mathrm{CT} 26$ tumor growth and increases cure rates compared to immunomodulation alone or DaRT alone}

In a previous investigation of the DA3 breast cancer mouse model, we showed that inhibition of immunosuppressive cells by the Treg inhibitor CP and the MDSC inhibitor sildenafil further enhances DaRT-induced primary/challenge tumor retardation and inhibits the development of lung metastases when used with or without $\mathrm{CpG}$ [13]. We therefore studied DaRT in combination with the immunomodulators $\mathrm{CpG}, \mathrm{CP}$, and sildenafil in the CT26 tumor model. CT26-bearing mice were treated as follows: 4 days prior to DaRT treatment, a systemic treatment with sildenafil was begun $(0.33 \mathrm{mg} / \mathrm{ml}$ in the drinking water, daily for 6 weeks), and an i.p. injection of $125 \mathrm{mg} / \mathrm{kg} \mathrm{CP}$ 
Fig. 1 CT26 tumor development following DaRT + TLR3 agonist. a Experimental scheme. CT26-bearing mice were treated with either a $40 \mathrm{kBq}$ DaRT seed or inert seed; 72 and $24 \mathrm{~h}$ prior to seed insertion, an intratumoral (i.t.) injection of $10 \mu \mathrm{g} / 30 \mu \mathrm{l}$ poly I:C or PBS was given. $\mathbf{b}$ Tumor growth curve. DaRT + poly I:C reduced tumor growth compared to all other groups. $p_{t \text { test }}<0.05$ for $\mathrm{DaRT}+$ poly I:C vs. control on days 3, 13 and 14, vs. DaRT alone on days $4-8$, and vs. poly I:C alone on days 3, 8-10, 12, 13

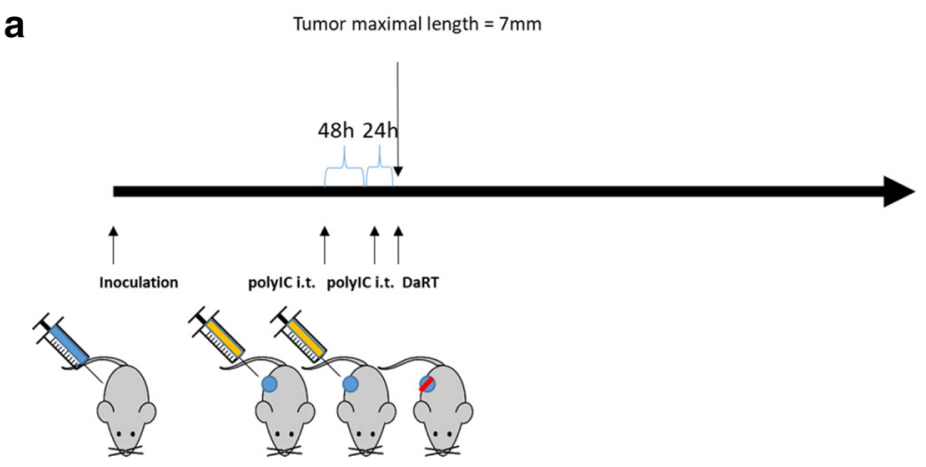

b

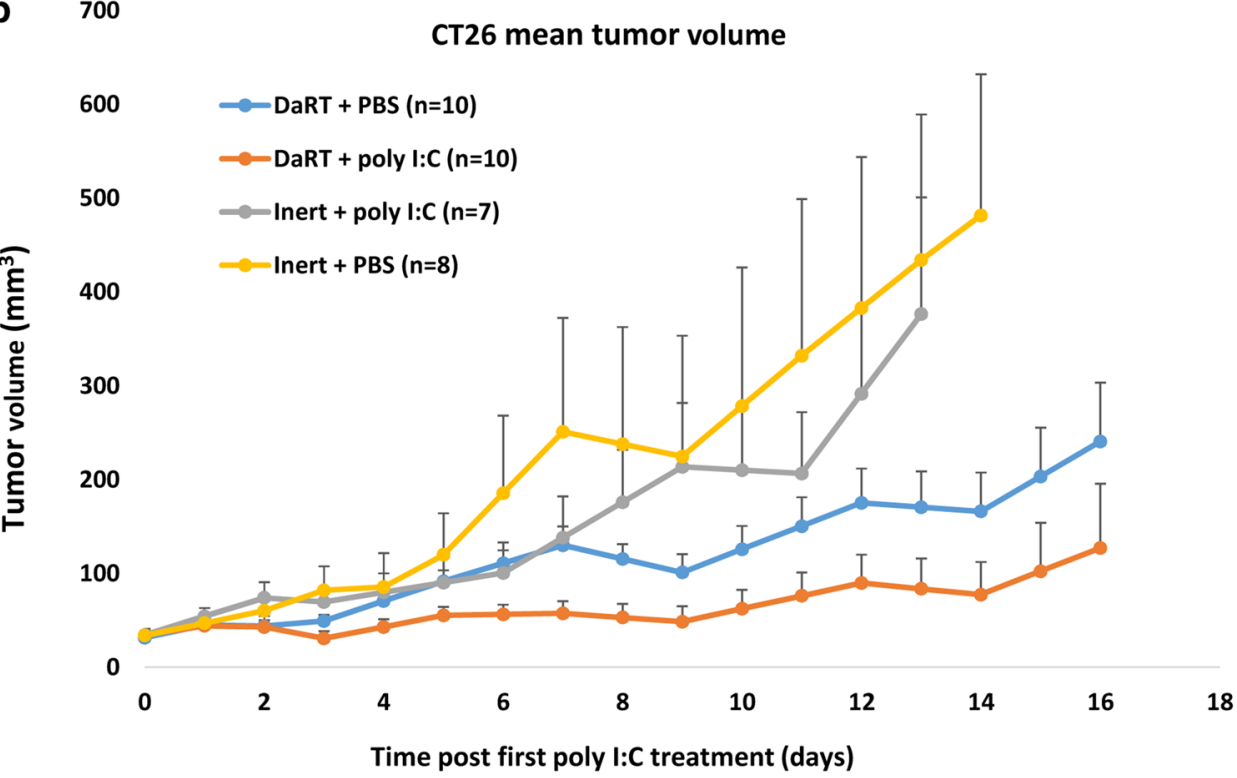

was given 1 day prior to DaRT. On the day of the DaRT treatment, $100 \mu \mathrm{g} / 30 \mu \mathrm{CpG}$ were administered peritumorally and three doses of $20 \mu \mathrm{g} / 10 \mu \mathrm{l} \mathrm{CpG}$ were given intranasally every 2 days thereafter (Fig. 3a). At this time point, maximal tumor length was $7-8 \mathrm{~mm}$. DaRT treatment was applied using a single $7-\mathrm{mm}{ }^{224} \mathrm{Ra}$ seed (60 kBq). One week after DaRT insertion an additional dose of CP was given (Fig. 3a).

Tumor-rejection rates were significantly enhanced in animals treated with the three immunomodulators in combination with DaRT seeds, compared to the same treatment with inert seeds or DaRT alone (Fig. 3b). In the group treated with DaRT alone, there were no cured tumors. For mice treated with immunomodulators and an inert seed, 16 out of 17 (94\% of the tumors) did not disappear or disappeared temporarily, whereas 22 out of 43 tumors (51\%) were cured in the group treated with DaRT combined with immunomodulators up to 93 days following treatment. The difference was significant $\left(P_{\chi^{2} \text { test }}<0.005\right)$ for DaRT combined with the three immunomodulators vs. all other groups (Fig. 3b). These results suggest that the immunomodulatory treatment and DaRT act synergistically to induce a long-term antitumor response.

In the group treated with DaRT and immunomodulators (Fig. 3b), 10\% of the tumors that had been cured relapsed on day 15 post-DaRT - the last day of systemic CpG treatment. We therefore tested whether an extension of the systemic intranasal $\mathrm{CpG}$ treatment would improve tumor retardation. In this experiment, mice were treated using the same treatment regime as described above, yet in one treatment group the systemic $\mathrm{CpG}$ treatment was extended ( 6 doses given every 2 days instead of 3 doses every 2 days). CT26-bearing mice were treated with $60-70 \mathrm{kBq}{ }^{224} \mathrm{Ra}$ DaRT seeds, combined with $100 \mu \mathrm{g} / 30 \mu \mathrm{l} \mathrm{CpG}$ injected peritumorally, three or six doses of $20 \mu \mathrm{g} / 10 \mu \mathrm{l} \mathrm{CpG}$ administered intranasally, $0.33 \mathrm{mg} / \mathrm{ml}$ sildenafil in the drinking water, and i.p. injection of $125 \mathrm{mg} / \mathrm{kg} \mathrm{CP}$. Inert seeds combined with immunomodulation that included the extended $\mathrm{CpG}$ treatment served as a control $(n=9)$. Corresponding with the results in Fig. 3, tumor-rejection rates in the group treated with DaRT and immunomodulators was $78 \%$ with the short $\mathrm{CpG}$ treatment, and $70 \%$ with the extended CpG treatment, vs. only $14 \%$ in 
Fig. 2 CT26 tumor rejection following DaRT combined with TLR9/TLR1,2 agonists. a Experimental scheme. CT26bearing mice were treated with a 40-50 kBq DaRT seed in combination with $\mathrm{CpG}$ or XS15. For XS15 treatment, a $40 \mu \mathrm{g} / 50 \mu \mathrm{l}$ peritumoral (p.t.) injection was given once a week for 3 weeks (three injections, first treatment on the day of DaRT insertion). For CpG treatment, a $100 \mu \mathrm{g} / 30 \mu \mathrm{l}$ p.t. injection was given immediately prior to DaRT insertion and $20 \mu \mathrm{g} / 10 \mu \mathrm{l}$ was administered intranasally (i.n.) three times thereafter, every 2 days. $\mathbf{b}$ Percent of tumor-bearing mice. $p_{\chi 2 \text { test }}<0.05$ for DaRT vs. DaRT + CpG. The presented results are based on cumulative data from two different experiments

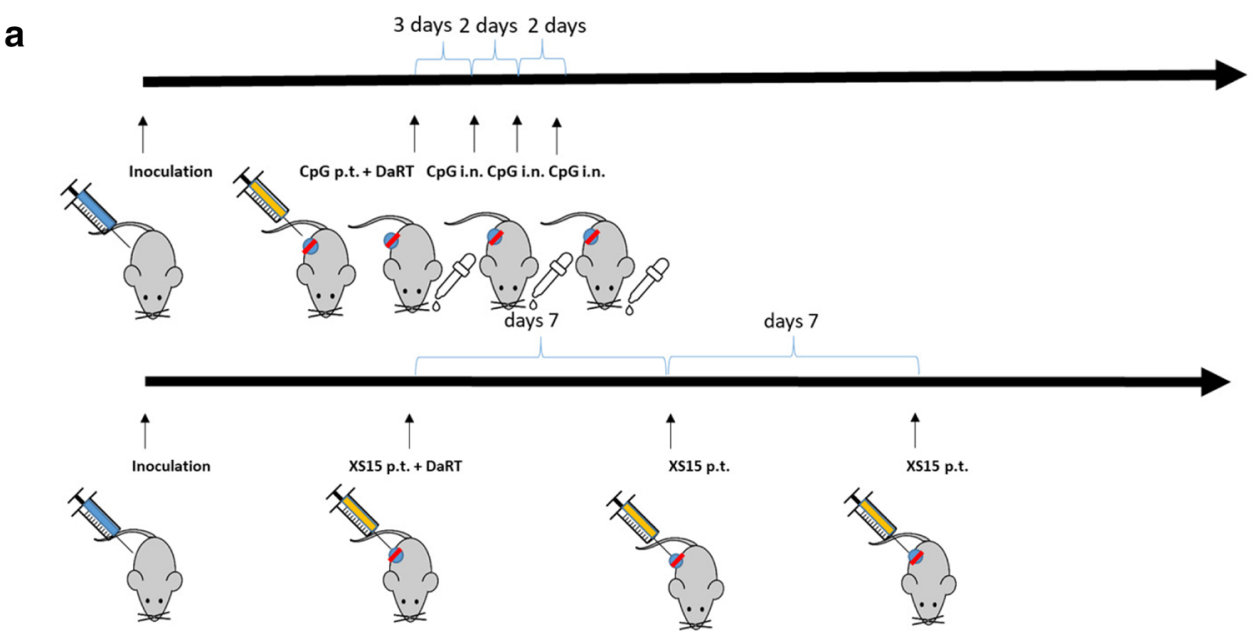

CT26 tumor-bearing mice

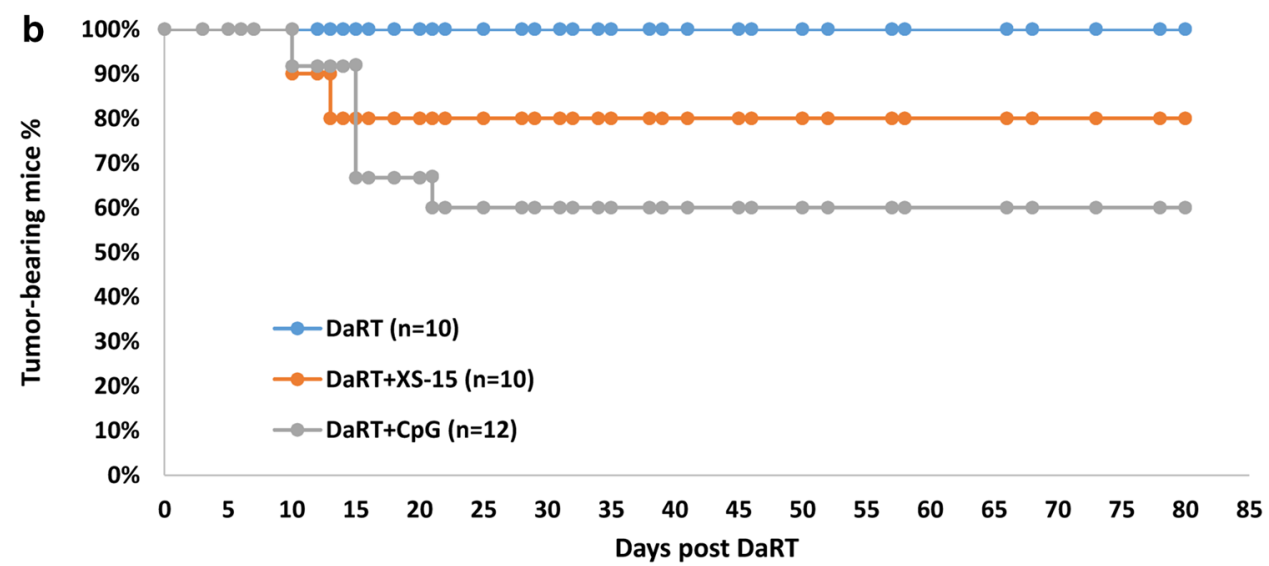

the control group (inert seed + immunomodulators). Thus, extension of the systemic $\mathrm{CpG}$ treatment at this time point did not significantly improve primary tumor retardation. Primary tumor volume (mean \pm SEM) on day 32 post-DaRT insertion was $90 \pm 81$ vs. $61 \pm 42.5 \mathrm{~mm}^{3}$ in the short $\mathrm{CpG}$ group ( $n=10)$ vs. the extended CpG group $(n=10)$, respectively $\left(p_{t \text { test }}>0.05\right)$.

\section{CT26-bearing mice cured by immunomodulation combined with DaRT, but not inert seed, show delayed tumor development when rechallenged with a higher number of cells}

The role of DaRT in inducing long-term antitumor immune memory when combined with immunomodulators $(\mathrm{CpG}$, sildenafil, and (P) was then investigated. Mice were treated by immunomodulation in combination with either DaRT or inert seed. Cured mice or mice that underwent tumor resection when tumor volume exceeded $150 \mathrm{~mm}^{3}$ were rechallenged with a higher number of cells $\left(5 \times 10^{6}\right.$ vs. $5 \times 10^{5}$ used in the initial inoculation) $\sim 4$ months after DaRT insertion.
An additional group of naïve mice served as controls. Mice that were treated with DaRT and the immunomodulators and then underwent tumor challenge, showed significantly retarded tumor growth compared to naïve mice inoculated with the same number of cells $\left(5 \times 10^{6} ; p_{t \text { test }}<0.05\right.$, on days 14-21). In contrast, retardation of tumor growth in mice treated with immunomodulators and inert seeds, compared to naïve mice, was not significant. Challenged tumor volume on day 21 postinoculation for mice originally treated with DaRT and immunomodulators $(n=13)$, the control group $(n=9)$ and naïve mice $(n=5)$ was $109 \pm 60,401 \pm 183$ and $951 \pm 247 \mathrm{~mm}^{3}$, respectively (Fig. 4).

\section{CT26-bearing mice cured by DaRT in combination with $C p G$, sildenafil and $C P$ resist tumor challenge via tumor-specific immune response}

The next question was whether the effect of the above combined treatment was due to a specific immune response against CT26 tumor antigens. Eighteen mice that were cured by DaRT and the three immunomodulators were challenged 


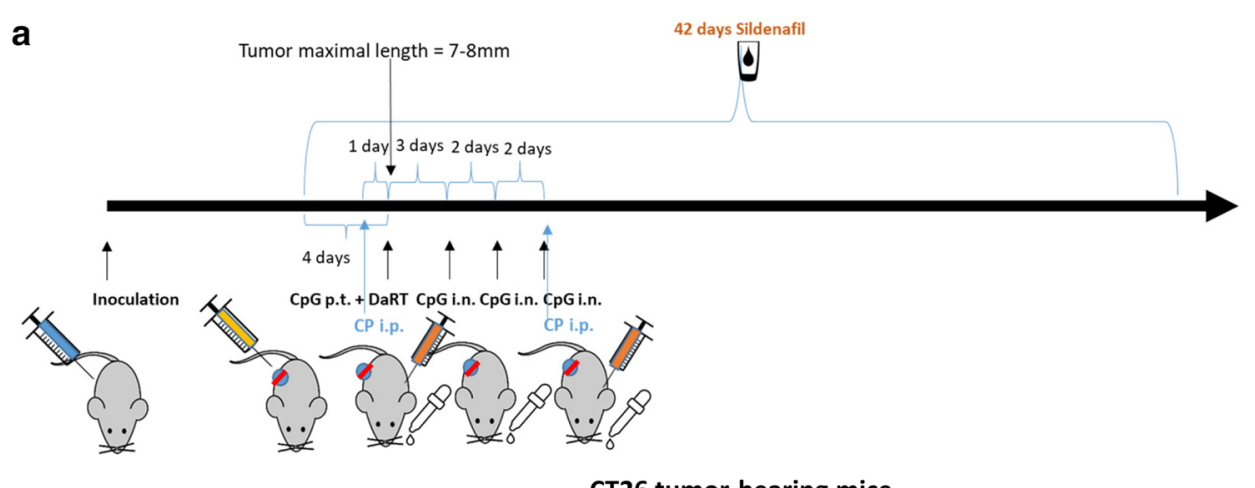

CT26 tumor-bearing mice

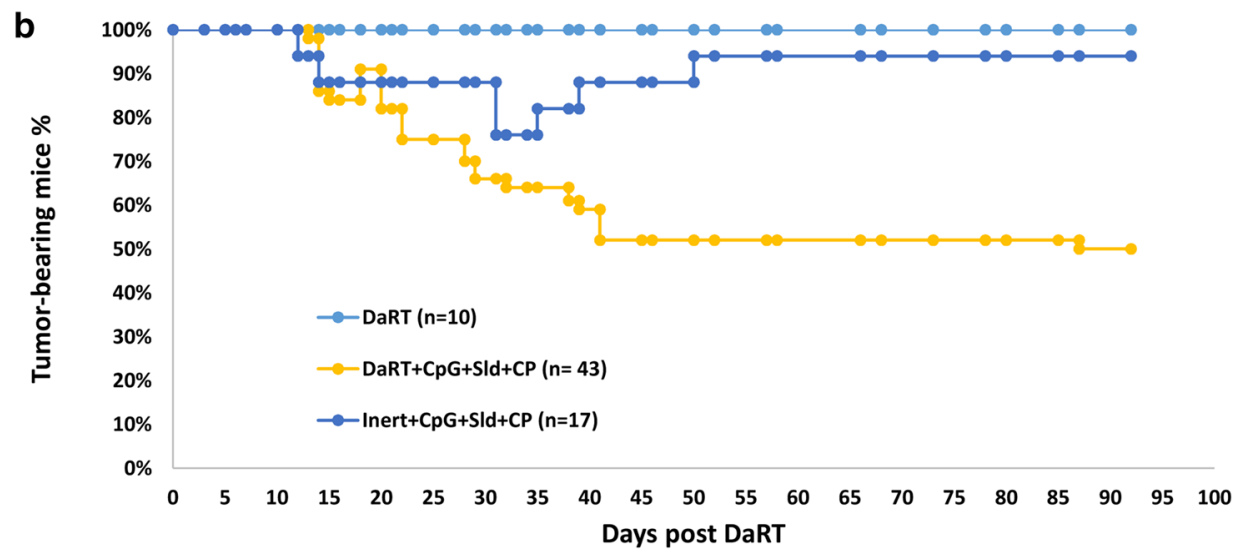

Fig. 3 CT26 tumor rejection following DaRT combined with TLR9 agonist and MDSC/Treg inhibitors. a Schematic representation of the treatment protocol combining DaRT with immunomodulators. Mice were inoculated with $5 \times 10^{5} \mathrm{CT} 26$ cells. When tumor maximal length reached 7-8 mm (10-14 days after inoculation), a single 7-mm $60 \mathrm{kBq}$ DaRT seed, or an inert seed, was inserted into the tumor. Four days prior to DaRT, systemic treatment with sildenafil was begun $(0.33 \mathrm{mg} / \mathrm{ml}$ in the drinking water, daily for 6 weeks $)$. An i.p. injec- tion of $125 \mathrm{mg} / \mathrm{kg} \mathrm{CP}$ was given 1 day before $\mathrm{DaRT}$ and 1 week after DaRT. On the day of DaRT treatment, $100 \mu \mathrm{g} / 30 \mu \mathrm{l} \mathrm{CpG}$ were administered peritumorally (p.t.) and three doses of $20 \mu \mathrm{g} / 10 \mu \mathrm{l} \mathrm{CpG}$ were given intranasally (i.n.) every 2 days starting from day 3 after DaRT. b Percent tumor-bearing mice during 3 months of follow-up. $p_{\chi 2 \text { test }}<0.005$ for DaRT combined with the three immunomodulators vs. all other groups. The presented results are based on cumulative data from three different experiments
Fig. 4 Rechallenge tumor development in CT26-bearing mice treated with DaRT vs. inert seed combined with immunomodulators. Mice were inoculated with $5 \times 10^{5}$ CT26 tumor cells and then treated with DaRT or inert seeds combined with immunomodulators $\mathrm{CpG}, \mathrm{CP}$, and sildenafil. Cured mice or tumorresected mice were rechallenged $\sim 4$ months after DaRT with $5 \times 10^{6}$ CT26 tumor cells

\section{CT26 challenge following the combined treatment}

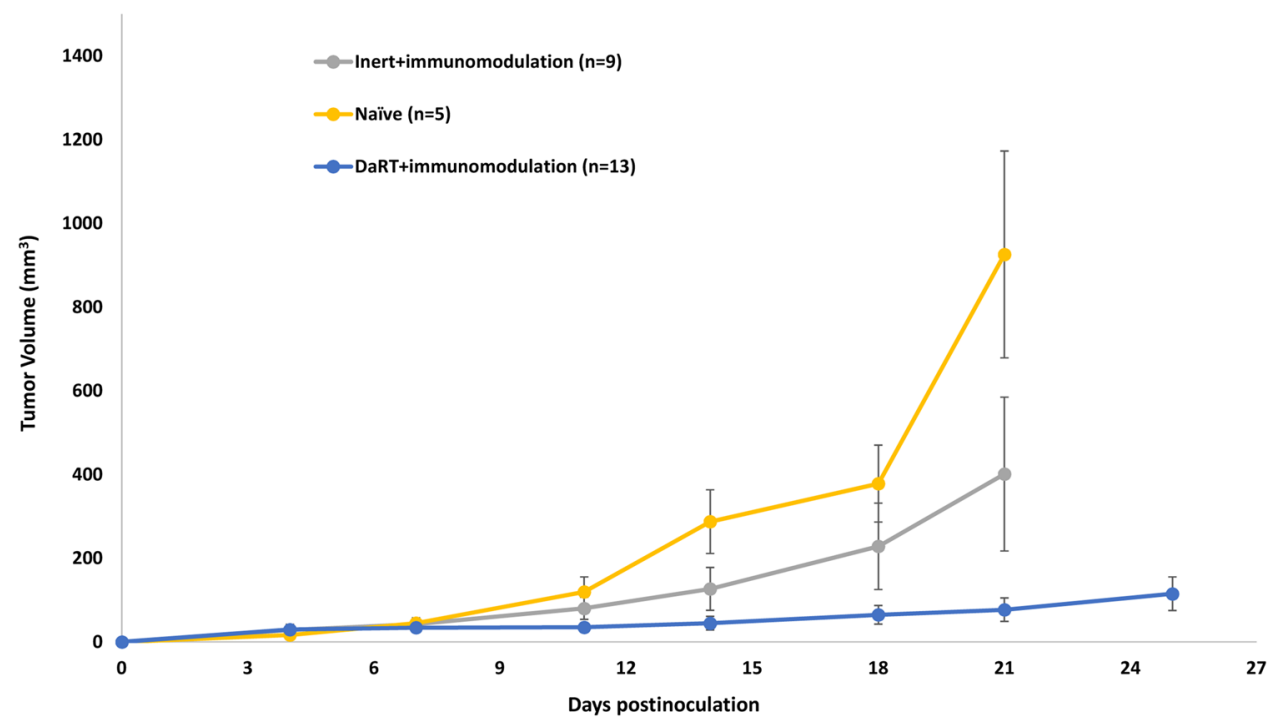


by an additional dose of the same amount of tumor cells $\left(5 \times 10^{5}\right)$ to their left flank. Ten animals were inoculated with CT26 tumor cells, and eight animals with DA3 tumor cells. Ten naïve mice served as controls. While none of the cured mice inoculated with CT26 cells developed tumors, all cured mice inoculated with DA3 did develop tumors (Table 1, Challenge assay).

In addition, the spleens of mice treated with DaRT + the immunomodulators were harvested 2-5 months after treatment and used to prepare a single-cell suspension. Thereafter, naïve mice were inoculated with DA3 or CT26 tumor cells that were mixed with the harvested splenocytes at 45:1 (splenocytes:tumor cells). Splenocytes of naïve mice served as a control. All mice inoculated with DA3 cells and all mice inoculated with CT26 cells in combination with naïve splenocytes developed tumors, whereas only $17 \%$ of the mice inoculated with CT26 cells and the splenocytes taken from mice that were previously treated with DaRT + immunomodulators developed tumors (Table 1, Winn assay). These results demonstrate that the immune response induced by the treatment of DaRT and immonomodulators is mediated by a specific long-term immune memory against CT26 antigens.

\section{Discussion}

The use of alpha radiation to treat solid tumors holds promise as an efficient, precise, and immunostimulating treatment of cancer. Due to the short range of this radiation's activity and its highly destructive capabilities, the treatment spares the healthy tissue surrounding the tumor on the one hand, and efficiently damages (dividing or non-dividing, normoxic or hypoxic) cells in the malignant tissue on the other. DaRT enables using this type of radiation locally due to its ability to expand the diffusion range of the radiation-emitting atoms, and thus to cover a therapeutically significant volume of the tumor. Importantly, the effect of the treatment is not only local, but also involves activation of a systemic immune response at distant sites [13].

In the present study, we have strengthened our results by employing the tumor-specific challenge and Winn assays. Protection of the mice against tumor cell reinoculation in the opposite lateral flank showed that the antitumor immune response is not only local. Moreover, we showed that DaRT was required for the induction of long-term tumor-specific immune memory and for long-lasting primary tumor rejection when combined with immunomodulation. This suggests a pivotal role for DaRT in the activation and cross-priming of antigen-presenting cells toward the induction of an antigen-specific T-cell response.

Treatment of DA3 breast adenocarcinoma tumors with DaRT in combination with the TLR9 agonist CpG retards local tumor growth, relative to DaRT alone or to $\mathrm{CpG}$ alone [13]. Consistent with these results, the current study confirmed that when combined, DaRT and CpG are the main components required for complete tumor rejection in CT26-bearing mice. We also showed that combining DaRT with additional types of TLR agonists similarly improves the antitumor effect. The interaction between TLR agonists and DaRT may be mediated by mechanisms

Table 1 Resistance to challenge of mice cured by DaRT + immunomodulators and specific protection of naïve mice treated with mouse splenocytes

\begin{tabular}{|c|c|c|c|c|c|}
\hline Challenge assay & Mouse source & $\begin{array}{l}\text { Challenge cell } \\
\text { line }\end{array}$ & $N$ & $\begin{array}{l}\text { Number of tumor- } \\
\text { bearing mice }\end{array}$ & $\begin{array}{l}\text { Tumor } \\
\text { development } \\
(\%)\end{array}$ \\
\hline & Cured by $\mathrm{DaRT}+\mathrm{CP}+\mathrm{CpG}+$ sildenafil & СТ26 & 10 & 0 & 0 \\
\hline & Naïve & CT26 & 10 & 10 & 100 \\
\hline & Cured by $\mathrm{DaRT}+\mathrm{CP}+\mathrm{CpG}+$ sildenafil & DA3 & 8 & 8 & 100 \\
\hline & Naïve & DA3 & 10 & 10 & 100 \\
\hline \multirow[t]{5}{*}{ Winn assay } & Splenocytes source & Cell line & $N$ & $\begin{array}{l}\text { Number of tumor- } \\
\text { bearing mice }\end{array}$ & $\begin{array}{l}\text { Tumor } \\
\text { development } \\
(\%)\end{array}$ \\
\hline & Treated by $\mathrm{DaRT}+\mathrm{CP}+\mathrm{CpG}+$ sildenafil & СТ26 & 12 & 2 & 17 \\
\hline & Naïve & СТ26 & 12 & 12 & 100 \\
\hline & Treated by $\mathrm{DaRT}+\mathrm{CP}+\mathrm{CpG}+$ sildenafil & DA3 & 9 & 9 & 100 \\
\hline & Naïve & DA3 & 8 & 8 & 100 \\
\hline
\end{tabular}

Mice that were cured by DaRT, sildenafil, low-dose CP and CpG $(n=18)$ and naïve mice $(n=20)$ were inoculated with $5 \times 10^{5}$ CT26 or DA3 cells (Challenge assay). Percent of animals that developed tumors following challenge is presented. Naïve mice were injected intradermally with splenocytes from either naïve or CT26-bearing mice treated by DaRT and immunomodulators, coupled with CT26 or DA3 tumor cells in the relation of $45 \mathrm{Ly}: 1 \mathrm{TC}$ (Winn assay). Percent of tumor development is presented. The presented results are based on cumulative data from two different experiments 
such as cross-presentation of tumor-associated neoantigens from dying cells, which are phagocytosed by dendritic cells [16-19], coupled with cytokine production and danger signals following TLR activation and DNA damage-induced cell death [19-24].

Extending the period of systemic $\mathrm{CpG}$ administration did not improve the treatment outcome, implying a limited period of activation or a response plateau. The question of tumor recurrence about 2 weeks after DaRT treatment warrants further study.

The response induced by DaRT and $\mathrm{CpG}$ was further enhanced by the inactivation of immune suppressor cellswhich impair antitumor activity - such as Tregs [25] and MDSCs [26]. Inhibition of these cells in combination with DaRT and immunoadjuvants resulted in a high percentage of cured animals, suggesting that even when danger signals and dying cells are present in the context of a pathogen, regulatory immune cells still inhibit the immune response. This is probably to protect from false-positive identification of selfantigens presented by cells in the healthy tissue surrounding the pathogen. However, immune adjuvant and inactivation of regulatory immune cells in the absence of DaRT resulted in tumor recurrence, suggesting that tumor ablation by DaRT promotes tumor antigen presentation, and serves to widen the tumor antigen repertoire recognized by the immune system. This is in agreement with previous reports demonstrating the enhancement of MHC1 presentation following radiation of tumor cells [27]. Indeed, this study showed that the cure achieved by the combined treatment was mediated by a specific immune response against tumor-associated antigens. The fact that this setup eliminated immunogenic CT26 tumors implies that it might also be effective with less immunogenic tumors following additional manipulation. For example, using strategies for additional enhancement of the upregulation of tumor-antigen presentation in the treated tumor, prior to the above described treatment.

Radiotherapy acts in synergy with immunostimulation to enhance the distant antitumor immune response [28], and several combinations of radiotherapy and immunotherapy have been tested in humans [28-32]. However, commonlyused types of radiation may cause adverse effects due to their wide range. Therefore, we may conclude that combining DaRT with immunotherapy provides three novel advantages in one treatment: (1) minor radiation-induced adverse effects due to short radiation range; (2) high radiation effectiveness; (3) strong synergy with immunotherapy that enhances the specificity and the distant effects of the antitumor immune response. Since the mortality rate of cancer patients depends mainly on the development of metastases [33], it is highly important to investigate strategies that boost the immune response induced by DaRT. Such strategies may provide a comprehensive treatment for both the local tumor and distant metastases, eventually leading to the possibility of a complete cure of the patients.

DaRT is currently being tested in clinical trials with squamous cell carcinoma patients and has demonstrated high tumor response rates without grade 3 or higher toxicity (NCT03353077). The development of strategies such as those presented here (i.e., tumor ablation by DaRT combined with immunoadjuvants and inhibitors of immunosuppressive cells) is required to move forward in the treatment of this challenging disease.

Acknowledgements This work was performed in partial fulfillment of the requirements toward an M.Sc. degree of Adi Cohen, Sackler Faculty of Medicine, Tel Aviv University.

Author Contributions YK and IK supervised the study; VD and YK wrote the manuscript; AC, ME, VD, and MS performed the experiments; AC, ME, and VD analyzed the data; SSN, AT and H-GR were involved in planning the experiments and editing the manuscript.

Funding This work was supported in part by a grant from the Israel Innovation Authority.

\section{Compliance with ethical standards}

Conflict of interest Yona Keisari and Itzhak Kelson serve as officers at Alpha Tau Medical Ltd. Tel Aviv, Israel. Hans-Georg Rammensee shares the patent for XS15. All other authors declare that they have no conflict of interest.

Ethical approval and ethical standards Experiments were performed in accordance with Israeli government and Tel Aviv University institute guidelines and regulations (Ethical committee permit no. 01-17-93).

Animal source Animals were obtained from Envigo (Jerusalem, Israel).

Cell line authentication The CT26 cell line is an N-nitroso-N-methylurethane (NNMU)-induced, undifferentiated colon carcinoma cell line that was purchased from the American Type Culture Collection (ATCC, CT26.WTATCC ${ }^{\circledR}$ CRL-2638 ${ }^{\mathrm{TM}}$ ) in 2015. Cells cryopreserved at their third passage following purchase were used for the experiments. The DA3 cell line is a dimethylbenzanthracene (DMBA)induced, undifferentiated breast adenocarcinoma cell line. The cell line was a gift from Prof. Ilan Tsarfati [34]. Both cell lines were tested for mycoplasma and were found to be mycoplasma free. Both cell lines were authenticated using short tandem repeat (STR) profiling (CellCheck $^{\mathrm{TM}}$, IDEXX BioAnalytics, USA). No mammalian interspecies contamination was detected. The STR DNA profile of DA3 cells was generated, for the first time, as follows: MCA-4-2: 21.3, 22.3; MCA5-5: 13, 14; MCA-6-4: 18, 20; MCA-6-7: 12, 13; MCA-9-2: 14, 15; MCA-12-1: 16; MCA-15-3: 22.3; MCA-18-3: 19, 20; MCA-X-1: 24.

Open Access This article is distributed under the terms of the Creative Commons Attribution 4.0 International License (http://creativeco mmons.org/licenses/by/4.0/), which permits unrestricted use, distribution, and reproduction in any medium, provided you give appropriate credit to the original author(s) and the source, provide a link to the Creative Commons license, and indicate if changes were made. 


\section{References}

1. Keisari Y (2017) Tumor abolition and antitumor immunostimulation by physico-chemical tumor ablation. Front Biosci (Landmark Ed) 22:310-347

2. Arazi L, Cooks T, Schmidt M, Keisari Y, Kelson I (2007) Treatment of solid tumors by interstitial release of recoiling shortlived alpha emitters. Phys Med Biol 52:5025-5042. https://doi. org/10.1088/0031-9155/52/16/021

3. Arazi L, Cooks T, Schmidt M, Keisari Y, Kelson I (2010) The treatment of solid tumors by alpha emitters released from (224) Ra-loaded sources-internal dosimetry analysis. Phys Med Biol 55:1203-1218. https://doi.org/10.1088/0031-9155/55/4/020

4. Cooks T, Arazi L, Efrati M, Schmidt M, Marshak G, Kelson I, Keisari Y (2009) Interstitial wires releasing diffusing alpha emitters combined with chemotherapy improved local tumor control and survival in squamous cell carcinoma-bearing mice. Cancer 115:1791-1801. https://doi.org/10.1002/cncr.24191

5. Cooks T, Arazi L, Schmidt M, Marshak G, Kelson I, Keisari Y (2008) Growth retardation and destruction of experimental squamous cell carcinoma by interstitial radioactive wires releasing diffusing alpha-emitting atoms. Int J Cancer 122:16571664. https://doi.org/10.1002/ijc. 23268

6. Cooks T, Schmidt M, Bittan H, Lazarov E, Arazi L, Kelson I, Keisari Y (2009) Local control of lung derived tumors by diffusing alpha-emitting atoms released from intratumoral wires loaded with radium-224. Int J Radiat Oncol Biol Phys 74:966973. https://doi.org/10.1016/j.ijrobp.2009.02.063

7. Cooks T, Tal M, Raab S et al (2012) Intratumoral 224Raloaded wires spread alpha-emitters inside solid human tumors in athymic mice achieving tumor control. Anticancer Res 32:5315-5321

8. Horev-Drori G, Cooks T, Bittan H, Lazarov E, Schmidt M, Arazi L, Efrati M, Kelson I, Keisari Y (2012) Local control of experimental malignant pancreatic tumors by treatment with a combination of chemotherapy and intratumoral 224radiumloaded wires releasing alpha-emitting atoms. Transl Res 159:32-41. https://doi.org/10.1016/j.trs1.2011.08.009

9. Carvalho HA, Villar RC (2018) Radiotherapy and immune response: the systemic effects of a local treatment. Clinics (Sao Paulo) 73:e557s. https://doi.org/10.6061/clinics/2018/e557s

10. Ogata T, Teshima T, Kagawa K et al (2005) Particle irradiation suppresses metastatic potential of cancer cells. Can Res 65:113-120

11. Zhuang Y, Li S, Wang H, Pi J, Xing Y, Li G (2018) PD-1 blockade enhances radio-immunotherapy efficacy in murine tumor models. J Cancer Res Clin Oncol 144:1909-1920. https://doi. org/10.1007/s00432-018-2723-4

12. Confino H, Hochman I, Efrati M, Schmidt M, Umansky V, Kelson I, Keisari Y (2015) Tumor ablation by intratumoral Ra-224-loaded wires induces anti-tumor immunity against experimental metastatic tumors. Cancer Immunol Immunother 64:191-199. https://doi.org/10.1007/s00262-014-1626-8

13. Confino H, Schmidt M, Efrati M, Hochman I, Umansky V, Kelson I, Keisari Y (2016) Inhibition of mouse breast adenocarcinoma growth by ablation with intratumoral alpha-irradiation combined with inhibitors of immunosuppression and CpG. Cancer Immunol Immunother 65:1149-1158. https://doi. org/10.1007/s00262-016-1878-6

14. Meyer C, Sevko A, Ramacher M et al (2011) Chronic inflammation promotes myeloid-derived suppressor cell activation blocking antitumor immunity in transgenic mouse melanoma model. Proc Natl Acad Sci USA 108:17111-17116. https://doi. org/10.1073/pnas.1108121108
15. Gouttefangeas C, Rammensee HG (2018) Personalized cancer vaccines: adjuvants are important, too. Cancer Immunol Immunother 67:1911-1918. https://doi.org/10.1007/s0026 2-018-2158-4

16. Sanchez-Paulete AR, Teijeira A, Cueto FJ, Garasa S, Perez-Gracia JL, Sanchez-Arraez A, Sancho D, Melero I (2017) Antigen cross-presentation and T-cell cross-priming in cancer immunology and immunotherapy. Ann Oncol 28:xii74. https://doi. org/10.1093/annonc/mdx727

17. Nakayama M (2014) Antigen presentation by MHC-dressed cells. Front Immunol 5:672. https://doi.org/10.3389/fimmu .2014 .00672

18. Jelinek I, Leonard JN, Price GE et al (2011) TLR3-specific double-stranded RNA oligonucleotide adjuvants induce dendritic cell cross-presentation, CTL responses, and antiviral protection. J Immunol 186:2422-2429. https://doi.org/10.4049/jimmu nol.1002845

19. Arnold KM, Flynn NJ, Raben A, Romak L, Yu Y, Dicker AP, Mourtada F, Sims-Mourtada J (2018) The impact of radiation on the tumor microenvironment: effect of dose and fractionation schedules. Cancer Growth Metastasis 11:1179064418761639. https://doi.org/10.1177/1179064418761639

20. Ozpiskin OM, Zhang L, Li JJ (2019) Immune targets in the tumor microenvironment treated by radiotherapy. Theranostics 9:1215-1231. https://doi.org/10.7150/thno.32648

21. Sanchez-Paulete AR, Teijeira A, Cueto FJ, Garasa S, Perez-Gracia JL, Sanchez-Arraez A, Sancho D, Melero I (2017) Antigen cross-presentation and T-cell cross-priming in cancer immunology and immunotherapy. Ann Oncol 28:xii44-xii55. https://doi. org/10.1093/annonc/mdx237

22. Yoshida S, Shime H, Takeda Y, Nam JM, Takashima K, Matsumoto M, Shirato H, Kasahara M, Seya T (2018) Toll-like receptor 3 signal augments radiation-induced tumor growth retardation in a murine model. Cancer Sci 109:956-965. https ://doi.org/10.1111/cas.13543

23. Scholch S, Rauber C, Weitz J, Koch M, Huber PE (2015) TLR activation and ionizing radiation induce strong immune responses against multiple tumor entities. Oncoimmunology 4:e1042201. https://doi.org/10.1080/2162402X.2015.1042201

24. Spiotto M, Fu YX, Weichselbaum RR (2016) The intersection of radiotherapy and immunotherapy: mechanisms and clinical implications. Sci Immunol 1:EAAG1266. https://doi. org/10.1126/sciimmunol.aag 1266

25. Shitara K, Nishikawa H (2018) Regulatory T cells: a potential target in cancer immunotherapy. Ann N Y Acad Sci 1417:104115. https://doi.org/10.1111/nyas.13625

26. Fleming V, Hu X, Weber R, Nagibin V, Groth C, Altevogt P, Utikal J, Umansky V (2018) Targeting myeloid-derived suppressor cells to bypass tumor-induced immunosuppression. Front Immunol 9:398. https://doi.org/10.3389/fimmu.2018.00398

27. Reits EA, Hodge JW, Herberts CA et al (2006) Radiation modulates the peptide repertoire, enhances MHC class I expression, and induces successful antitumor immunotherapy. J Exp Med 203:1259-1271. https://doi.org/10.1084/jem.20052494

28. Marabelle A, Kohrt H, Caux C, Levy R (2014) Intratumoral immunization: a new paradigm for cancer therapy. Clin Cancer Res 20:1747-1756. https://doi.org/10.1158/1078-0432. CCR-13-2116

29. Aznar MA, Tinari N, Rullan AJ, Sanchez-Paulete AR, Rodriguez-Ruiz ME, Melero I (2017) Intratumoral delivery of immunotherapy-act locally, think globally. J Immunol 198:31-39. https://doi.org/10.4049/jimmunol.1601145

30. Shekarian T, Valsesia-Wittmann S, Brody J, Michallet MC, Depil S, Caux C, Marabelle A (2017) Pattern recognition receptors: immune targets to enhance cancer immunotherapy. Ann Oncol 28:1756-1766. https://doi.org/10.1093/annonc/mdx179 
31. Wang Y, Deng W, Li N, Neri S, Sharma A, Jiang W, Lin SH (2018) Combining immunotherapy and radiotherapy for cancer treatment: current challenges and future directions. Front Pharmacol 9:185. https://doi.org/10.3389/fphar.2018.00185

32. Van Limbergen EJ, De Ruysscher DK, Olivo Pimentel V et al (2017) Combining radiotherapy with immunotherapy: the past, the present and the future. Br J Radiol 90:20170157. https://doi. org/10.1259/bjr.20170157

33. Guan X (2015) Cancer metastases: challenges and opportunities. Acta Pharm Sin B 5:402-418. https://doi.org/10.1016/j. apsb.2015.07.005

34. Leibowitz-Amit R, Tsarfaty G, Abargil Y, Yerushalmi GM, Horev J, Tsarfaty I (2006) Mimp, a mitochondrial carrier homologue, inhibits Met-HGF/SF-induced scattering and tumorigenicity by altering Met-HGF/SF signaling pathways. Can Res 66:8687-8697. https://doi.org/10.1158/0008-5472. CAN-05-2294

Publisher's Note Springer Nature remains neutral with regard to jurisdictional claims in published maps and institutional affiliations.

\section{Affiliations}

\section{Vered Domankevich ${ }^{1} \cdot$ Adi Cohen ${ }^{1} \cdot$ Margalit Efrati $^{1} \cdot$ Michael Schmidt $^{2,3} \cdot$ Hans-Georg Rammensee ${ }^{4}$. Sujit S. Nair ${ }^{5}$. Ashutosh Tewari $^{5} \cdot$ Itzhak Kelson $^{2,3} \cdot$ Yona Keisari $^{1,3}$}

1 Department of Clinical Microbiology and Immunology, Sackler Faculty of Medicine, Tel Aviv University, P.O. Box 39040, 6997801 Tel Aviv, Israel

2 School of Physics and Astronomy, Sackler Faculty of Exact Sciences, Tel Aviv University, Tel Aviv, Israel

3 Alpha Tau Medical, Tel Aviv, Israel
4 Department of Immunology, Institute for Cell Biology, University of Tübingen, Tübingen, Germany

5 Department of Urology, Icahn School of Medicine at Mount Sinai, New York, NY, USA 